\title{
Can libration maintain Enceladus's ocean?
}

\author{
Alec Wilson ${ }^{1} \&$ Rich Kerswell ${ }^{1,2}$ \\ ${ }^{1}$ School of Mathematics, University of Bristol, Bristol, BS8 1TW, UK \\ ${ }^{2}$ DAMTP, Centre for Mathematical Sciences, University of Cambridge, Cambridge,CB3 \\ $O W A, U K$
}

\begin{abstract}
The process by which the subsurface ocean on Enceladus is heated remains a puzzle. Tidal interaction with Saturn and Dione is the leading candidate but whether the dominant heating occurs in the solid core, ice crust or in the ocean itself is an outstanding question. Here we consider the driving effect of the longitudinal libration of the ice crust on the subsurface ocean and argue that the flow response should be turbulent even in the most benign situation of a smooth spherical ice shell when the motion of the boundary is only transmitted viscously. A rigorous upper bound on the turbulent viscous dissipation rate is then derived and used to argue that libration should be potent enough to explain the observed heat flux emanating out of Enceladus when the effects of tidal distortion and roughness of the ice crust are included.

Keywords: dissipation, libration, turbulence
\end{abstract}

\section{Introduction}

The data from recent solar system probes suggest the existence of subsurface oceans in many icy satellites [1] which opens up the fascinating possibility of extraterrestrial life relatively close by. Of the many oceans currently believed 5 to exist, those in Europa, Titan and Enceladus are the most certain having been inferred by more than one type of measurement (e.g. for Enceladus see [2] ). Given the surface temperatures in the outer solar system, there is a question as to how these oceans avoid freezing and answering this is nowhere more

Preprint submitted to Earth and Planetary Science Letters

July 9, 2018 
challenging than on Enceladus given its small size (its radius is just $252 \mathrm{~km}$ ) has an orbital eccentricity of 0.0047 with current observations suggesting that Enceladus's ice crust is decoupled from its rocky core: i.e. there is a global subsurface ocean $[2,8,9$. The question is then where the dominant tidal heating occurs - is it in the rocky core, the ice-crust or in the ocean itself? they induce is well understood and established after the successful prediction of Io's volcanic state [10, 11. As a result, the majority of studies have focussed either on Enceladus's solid core [12, 13, 14, 15, 16] or, more recently, on its ice crust [5, 6, 17. Despite this work, no generally-accepted solid model for tidally heating Enceladus's ocean has emerged [1].

In contrast, the ability of tidal effects to drive fluid flows and thereby heat the ocean itself through the accompanying viscous dissipation is poorly understood. Dissecting the exact tidal response of the coupled system of solid core, subsurface ocean and overlying ice crust is a complicated problem fraught with unknowns. passive flexible skin over a tidal ocean and the counter scenario b) where the ice crust is rigid enough to suppress ocean tides but which instead drives the ocean through its libration. An attraction of the latter is that it replaces the tidal body force on the ocean by a boundary condition forcing which is conducive to 35 laboratory modelling. The former limiting scenario was first considered by Tyler [18, 19, 20, 21] who used Laplace's 2D tidal equations for a shallow ocean to show via a resonant response that more than enough energy could be deposited in Enceladus's ocean given a large enough obliquity angle or a shallow enough ocean (see also [22, 23] and 24] who claim that this is not the case for a non- 
resonant response).

Concurrently another body of work has considered the second limiting scenario by studying the effect of imposing a longitudinal libration on rotating fluid-filled spheres, spherical shells and ellipsoids [25, 26, 27, 28, 29, 30]. Here the approach has been a combination of laboratory and numerical experiments treating the full 3D Navier-Stokes equations. While the use of tidally-distorted containers is more realistic for planetary applications, librating axisymmetric containers are (far) easier to theoretically model and allow the simpler and weaker effect of only (viscous) tangential forcing to be understood. In this vein, the experiments of Noir et al. 25. librate only spherical shells yet even then the flow clearly becomes turbulent near the boundary at sufficient librational amplitude. Later numerical work [31] showed that inertial waves could be driven into the interior which, when driven at sufficient amplitude, should break down to small scale turbulence through triad resonances [32] as seen in experiments with ellipsoidal containers [30]. No estimates of the turbulent viscous heating have, however, emerged for either the axisymmetric or asymmetric container situations as yet due either to the difficulty of measuring it in the laboratory or resolving all the relevant length scales on the computer.

The purpose of this paper is remedy this situation at least in the axisymmetric situation by theoretically deriving a rigorous upper bound on the turbulent dissipation rate inside a librating fluid-filled spherical shell directly from the governing Navier-Stokes equations. The result makes a prediction for the scaling of the dissipation rate with the parameters of the problem (the libration amplitude, the libration frequency and Ekman number) in the limit of vanishing Ekman number. The numerical prefactor, however, is typically very conservative and data - either from laboratory experiments or numerical simulations - are needed to renormalise the bound down to make a realistic prediction of what dissipation actually occurs. Exactly this approach was used to estimate the turbulent dissipation rate possible in a precessing spheroid [33, 34. There the bound correctly predicts that the dissipation rate becomes independent of the precession rate and Ekman number but the numerical prefactor needs to be renormalised 
by one and a half orders of magnitude to match experimental data (see figure 1 in [34). Significantly, even with this adjustment, this bound highlights the fact that precessionally-driven turbulence in the outer core is energetic enough to drive the geodynamo 34 .

That the response to Enceladus's (longitudinal) libration should be turbulent even under the idealised assumption of a smooth axisymmetric ice crust-ocean interface is clear from experiments [25] (see figure 1) and a simple estimate. The situation below the librating ice crust is essentially Stokes's second problem where an oscillating plane drives a half space of fluid. For Enceladus, the

80 Reynolds number can be estimated as $R o / \sqrt{E} \approx 7200$ where $R o \approx 2.1 \times 10^{-3}$ is ratio of the peak librational rotation rate to the basic spin rate (the Rossby number) and the Ekman number $E \approx 8.5 \times 10^{-14}$ (see Table 1). This is well above the Reynolds number of $\approx 500$ for which the flow to observed to be turbulent [35. [1]

\section{2. Bounding the dissipation in a librating spherical shell}

Enceladus's subsurface ocean is modelled as a fluid-filled layer sandwiched between two concentric hard spheres of inner and outer radii $r=r_{i}$ and $r_{o}$ respectively. For simplicity, the inner sphere is assumed to be uniformly rotating about its axis (labelled hereafter as the $z$-axis) at a constant angular frequency $\Omega$ (i.e. we ignore the libration of Enceladus's solid core which is estimated as being 4 times smaller than that of the ice crust: see Table 1 in [2]) whilst the outer sphere is librating (so rotating about the same axis at $\Omega(1+R o \sin \omega t$ ) where the Rossby number, $R o$, is the amplitude (in units of the basic rotation rate $\Omega$ ) and $\omega$ the angular frequency of the libration). Non-dimensionalising using the outer radius $r_{o}$ and the base rotation rate $\Omega$, the Navier-Stokes equations in the

\footnotetext{
${ }^{1}$ Note that the Reynolds number in $[35$ is $\sqrt{2}$ larger than the one used here.
} 
librating frame of the outer boundary $2^{2}$ are then

$$
\frac{\partial \boldsymbol{u}}{\partial t}+\boldsymbol{u} \cdot \nabla \boldsymbol{u}+2[1+\gamma(t)] \hat{\boldsymbol{z}} \times \boldsymbol{u}+\dot{\gamma}(t) \hat{\boldsymbol{z}} \times \boldsymbol{r}+\nabla p=2 E \nabla \cdot \boldsymbol{e},
$$

where $\gamma(t):=\operatorname{Rosin}\left(f^{*} t\right)$ with $f^{*}:=\omega / \Omega, \dot{\gamma}:=d \gamma / d t, e_{i j}:=\frac{1}{2}\left(\partial_{j} u_{i}+\partial_{i} u_{j}\right)$, the Ekman number $E:=\nu / r_{o}^{2} \Omega$ ( $\nu$ is the kinematic viscosity) and the fluid is assumed incompressible

$$
\nabla \cdot \boldsymbol{u}=0
$$

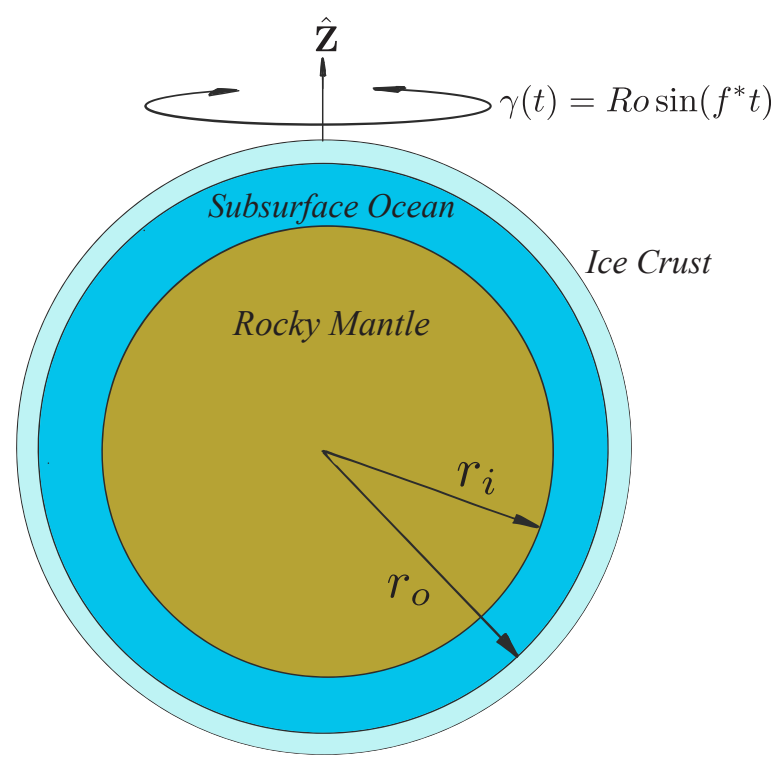

Figure 1: A model of librating Enceladus. The picture is in the mantle frame which is presumed uniformly rotating. In this the crust is librating across the top of the subsurface ocean (inner radius $r_{i}$ and outer radius $r_{o}$ ). Figure courtesy of Jerome Noir.

In this librating frame the outer boundary is stationary so $\left.\boldsymbol{u}\right|_{r=1}=\mathbf{0}$ while the inner boundary appears to oscillate back and forth with velocity $\left.\boldsymbol{u}\right|_{r=1-d}=$ $-\gamma(t) \hat{\boldsymbol{z}} \times \boldsymbol{r}$ where $d=\left(r_{o}-r_{i}\right) / r_{o}$ is the non-dimensional ocean depth. The (non-dimensionalised) long-time-averaged energy dissipation rate per unit mass

\footnotetext{
${ }^{2}$ We use this frame to demonstrate the general situation although for the particular set up here, a frame fixed in the inner boundary would be easier to analyse.
} 
is defined as

$$
\varepsilon:=2 E\left\langle\frac{1}{\mathcal{V}} \iiint_{V} e_{i j} e_{i j} \mathrm{dV}\right\rangle
$$

105

$$
\begin{aligned}
-\left\langle\dot{\gamma} \int_{r^{\prime}}^{1} \oint \boldsymbol{u} \cdot \hat{\boldsymbol{z}} \times \boldsymbol{r} d S d r\right\rangle+2\left\langle\gamma(1+\gamma) \int_{r^{\prime}}^{1} \oint \nabla \cdot\left(\frac{1}{2} s^{2} \boldsymbol{u}\right) d S d r\right\rangle \\
-\left\langle\gamma \oint_{r=r^{\prime}} s u w d S\right\rangle=2 E\left\langle\gamma\left[\oint_{r=1} s e_{r \phi} d S-\oint_{r=r^{\prime}} s e_{r \phi} d S\right]\right\rangle
\end{aligned}
$$

Here, the first term is the $\gamma$-rectified rate of change of the angular momentum in the shell (since $\left\langle\gamma(t) s \hat{\boldsymbol{\phi}} \cdot \frac{\partial \boldsymbol{u}}{\partial t}\right\rangle=-\langle\boldsymbol{u} \cdot \dot{\gamma} \hat{\boldsymbol{z}} \times \boldsymbol{r}\rangle$ ) or perhaps more understandably one part of the rate of change of rotational kinetic energy which vanishes on time-averaging

$$
\left\langle\frac{d}{d t}(\gamma \hat{\boldsymbol{z}} \cdot \boldsymbol{r} \times \boldsymbol{u})\right\rangle=\left\langle\dot{\gamma} \hat{\boldsymbol{z}} \cdot \boldsymbol{r} \times \boldsymbol{u}+\gamma \hat{\boldsymbol{z}} \cdot \boldsymbol{r} \times \frac{\partial \boldsymbol{u}}{\partial t}\right\rangle=0 .
$$

${ }_{125}$ The second term comes from the Coriolis torque, the third comes from the nonlinearity of the Navier-Stokes equations and the fourth term is the boundary 
torque contribution. The special choice $r^{\prime}=1-d$ causes both the Coriolis and nonlinear terms to vanish (no flow across the inner surface) and gives simply

$$
\mathcal{V} \varepsilon=2 E\left\langle\left.\gamma \oint_{r=1} s e_{r \phi}\right|_{r=1} d S\right\rangle
$$

after utilising (4). With this, (5) can be rearranged to give

$$
\begin{aligned}
\varepsilon= & -\left\langle\frac{\dot{\gamma}}{\mathcal{V}} \int_{r^{\prime}}^{1} \oint \boldsymbol{u} \cdot \hat{\boldsymbol{z}} \times \boldsymbol{r} d S d r\right\rangle-\left\langle\frac{\gamma}{\mathcal{V}} \oint_{r=r^{\prime}} s u w d S\right\rangle \\
& +2\left\langle\frac{\gamma(1+\gamma)}{\mathcal{V}} \int_{r^{\prime}}^{1} \oint \nabla \cdot\left(\frac{1}{2} s^{2} \boldsymbol{u}\right) d S d r\right\rangle+2 E\left\langle\frac{\gamma}{\mathcal{V}} \oint_{r=r^{\prime}} s e_{r \phi} d S\right\rangle
\end{aligned}
$$

130 The final step is to average 8 over the layer $r^{\prime} \in[1-\ell, 1]$ - i.e. form $\frac{1}{\ell} \int_{1-\ell}^{1} 8 d r^{\prime}$ - where $0<\ell<d$ is a free variable to be selected judiciously later. Then taking the modulus of each of the terms yields the following simple bound on the dissipation

$$
\begin{aligned}
\varepsilon \leqslant & \left|\left\langle\frac{\dot{\gamma}}{\mathcal{V}} \frac{1}{\ell} \int_{1-\ell}^{1} \int_{r^{\prime}}^{1} \oint \boldsymbol{u} \cdot \hat{\boldsymbol{z}} \times \boldsymbol{r} d S d r d r^{\prime}\right\rangle\right|^{(1)} \\
& +\left|\left\langle\frac{\gamma}{\mathcal{V}} \frac{1}{\ell} \int_{1-\ell}^{1} \oint s u w d S d r^{\prime}\right\rangle\right|^{(2)} \\
& +2\left|\left\langle\frac{\gamma(1+\gamma)}{\mathcal{V}} \frac{1}{\ell} \int_{1-\ell}^{1} \int_{r^{\prime}}^{1} \oint \nabla \cdot\left(\frac{1}{2} s^{2} \boldsymbol{u}\right) d S d r d r^{\prime}\right\rangle\right|^{(3)} \\
& +2 E\left|\left\langle\frac{\gamma}{\mathcal{V}} \frac{1}{\ell} \int_{1-\ell}^{1} \oint_{r=r^{\prime}} s e_{r \phi} d S d r^{\prime}\right\rangle\right|^{(4)} .
\end{aligned}
$$

The strategy now to is bound each of these terms by some appropriate function

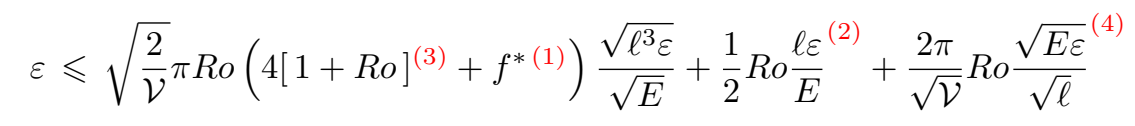

where the red superscripts indicate the origin of the terms from (9). Minimising this expression over $\ell$ yields an optimal value of $\ell=\left(4 \pi^{2} / \mathcal{V}\right)^{1 / 3} E \varepsilon^{-1 / 3}$ when the second and third terms contribute at leading order and the first is negligible in the limit $E \rightarrow 0$ (which is why the particular frame of reference is unimportant) and the best (lowest) bound

$$
\varepsilon \leqslant \frac{27 \pi^{2}}{2} \frac{R o^{3}}{\mathcal{V}}
$$


where $\mathcal{V}=4 \pi\left(1-(1-d)^{3}\right) / 3$ is the non-dimensional volume (with this maximal value of $\varepsilon$, the optimal boundary layer scale is $O(E / R o)$ and so smaller than the usual Ekman boundary layer scale of $O(\sqrt{E}))$. It turns out that $d=1$ can be taken in this result to retrieve a bound for a librating fluid-filled sphere. Past experience in other bounding calculations suggests that the numerical prefactor in the bound (11) could be improved (decreased) by an order of magnitude by solving the variational problem numerically (e.g. see expressions (2.43) and (2.55) in [34]). After this, one would then still expect the bound to be about 1.5 orders of magnitude above the data (e.g. see figure 1 in [34]) giving a likely total renormalisation factor of $10^{2.5} \approx 300$ of the bound down to produce a realistic numerical prediction of the turbulent dissipation rate. It's worth emphasizing that in the turbulent regime, this renormalisation only needs to be done once by its very nature the numerical prefactor does not depend on the parameters of the problem (i.e. it is not $E$-dependent).

\section{Implications}

The bound (11) is on the turbulent dissipation rate per unit mass (of fluid) in units of $r_{o}^{2} \Omega^{3}$. A bound on the total (dimensional) dissipation rate is therefore

$$
\varepsilon \leqslant \frac{27 \pi^{2}}{2} R o^{3} \rho \Omega^{3} r_{o}^{5}
$$

which formalises the usual estimate of the turbulent dissipation rate as the rate of working of turbulent drag (parametrised by a drag coefficient $c_{D}$ ) integrated over the (outer) boundary: i.e. $\rho c_{D} u^{2} \times u \times 4 \pi r_{o}^{2}=4 \pi c_{D} \rho u^{3} r_{o}^{2}$ where $u$ is estimated as $(\Omega R o) r_{o}$. The bound translates into the condition that $c_{D} \leqslant$ $27 \pi / 8 \approx 10.6$ or, using a renormalisation factor of $10^{2.5}$, that $c_{D} \approx 0.0335$.

The dimensional dissipation rate bound per unit surface area of the outer ice shell (of thickness $d_{i} r_{o}$ ) is

$$
\varepsilon^{*} \leqslant \varepsilon_{\text {bound }}^{*}:=\frac{27 \pi^{2}}{2} \frac{\rho\left(\operatorname{Ror}_{o} \Omega\right)^{3}}{4 \pi\left(1+d_{i}\right)^{2}},
$$

and $\rho$ is the density of the fluid. For Enceladus, the radius of the rocky core is inferred to be $180-185 \mathrm{~km}\left(=r_{o}(1-d)\right)$, the mean total radius is $252 \mathrm{~km}$ 
$\left(=r_{o}\left(1+d_{i}\right)\right)[2]$ and the ice shell thickness is $18-22 \mathrm{~km}\left(=d_{i} r_{o}\right)$ on average [37. The base rotation rate $\Omega$ is $2 \pi /(1.4$ days $) \approx 5.2 \times 10^{-5} \mathrm{~s}^{-1}$, the body

librates at the same frequency as the base rotation so $f^{*}=1[2]$ and the libration magnitude is $0.12^{\circ}$, so $R o=2.1 \times 10^{-3}$. Using these values gives a bound on the dissipation per unit surface area of Enceladus

$$
\epsilon^{*} \leqslant \frac{27 \pi}{8} \frac{\left(2.1 \times 10^{-3}\right)^{3}\left(232 \times 10^{3}\right)^{5}\left(5.2 \times 10^{-5}\right)^{3} \times 10^{3}}{\left(252 \times 10^{3}\right)^{2}} \mathrm{~W} / \mathrm{m}^{2} \approx 146 \times 10^{-3} \mathrm{~W} / \mathrm{m}^{2}
$$

which is 20 times greater than the measured value of the globally averaged flux of $7 \times 10^{-3} \mathrm{~W} / \mathrm{m}^{2}[20]$.

\begin{tabular}{lllllllll}
\hline Planet & Interior & $r_{o}(\mathrm{~km})$ & $r_{i}(\mathrm{~km})$ & $T_{\text {spin }}($ day $)$ & $f^{*}$ & $E$ & $R o$ & $R e$ \\
\hline Callisto & SO & $\sim 2300$ & $2000-2300$ & 16.68 & 1 & $4 \times 10^{-14}$ & $4.22 \times 10^{-6}$ & $\sim 20$ \\
Ganymede & LC & $\sim 800$ & $0-500$ & 7.15 & 1 & $4 \times 10^{-14}$ & $5.64 \times 10^{-6}$ & $\sim 25$ \\
Earth's moon & LC & $\sim 350$ & $0-150$ & 27.3 & 1 & $10^{-12}$ & $7 \times 10^{-5}$ & $\sim 73$ \\
Titan(Grav) & SO & $\sim 2500$ & $2350-2450$ & 15.95 & 1 & $3.5 \times 10^{-14}$ & $2.3 \times 10^{-5}$ & $\sim 123$ \\
Mercury & LC & $\sim 1800$ & $0-1700$ & 58.6 & $2 / 3$ & $7.5 \times 10^{-14}$ & $1.33 \times 10^{-4}$ & $\sim 490$ \\
Titan(Atm) & SO & $\sim 2500$ & $2350-2450$ & 15.95 & $3 \times 10^{-3}$ & $3.5 \times 10^{-14}$ & $1.3 \times 10^{-4}$ & $\sim 690$ \\
Io & LC & $\sim 500$ & - & 1.77 & 1 & $3 \times 10^{-14}$ & $1.3 \times 10^{-4}$ & $\sim 800$ \\
Europa & SO & $\sim 1450$ & $1300-1400$ & 3.55 & 1 & $2.3 \times 10^{-14}$ & $2 \times 10^{-4}$ & $\sim 1300$ \\
Enceladus & SO & $\sim 233$ & $180-185$ & 1.4 & 1 & $8.5 \times 10^{-14}$ & $2.1 \times 10^{-3}$ & $\sim 7200$ \\
Pluto & SO & $\sim 975$ & 830 & 6.39 & 1 & $9.2 \times 10^{-14}$ & $2.04 \times 10^{-5}$ & $\sim 67$ \\
\hline
\end{tabular}

Table 1: A reproduction and update of table 2 of 25] (see original table for their sources) which uses viscosities $\nu=10^{-6}$ and $\nu=3 \times 10^{-7} \mathrm{~m}^{2} / \mathrm{s}$, respectively, for subsurface oceans (SO) and liquid cores (LC). The data for Enceladus [2, 37] and Pluto [3, 38] have been added. 


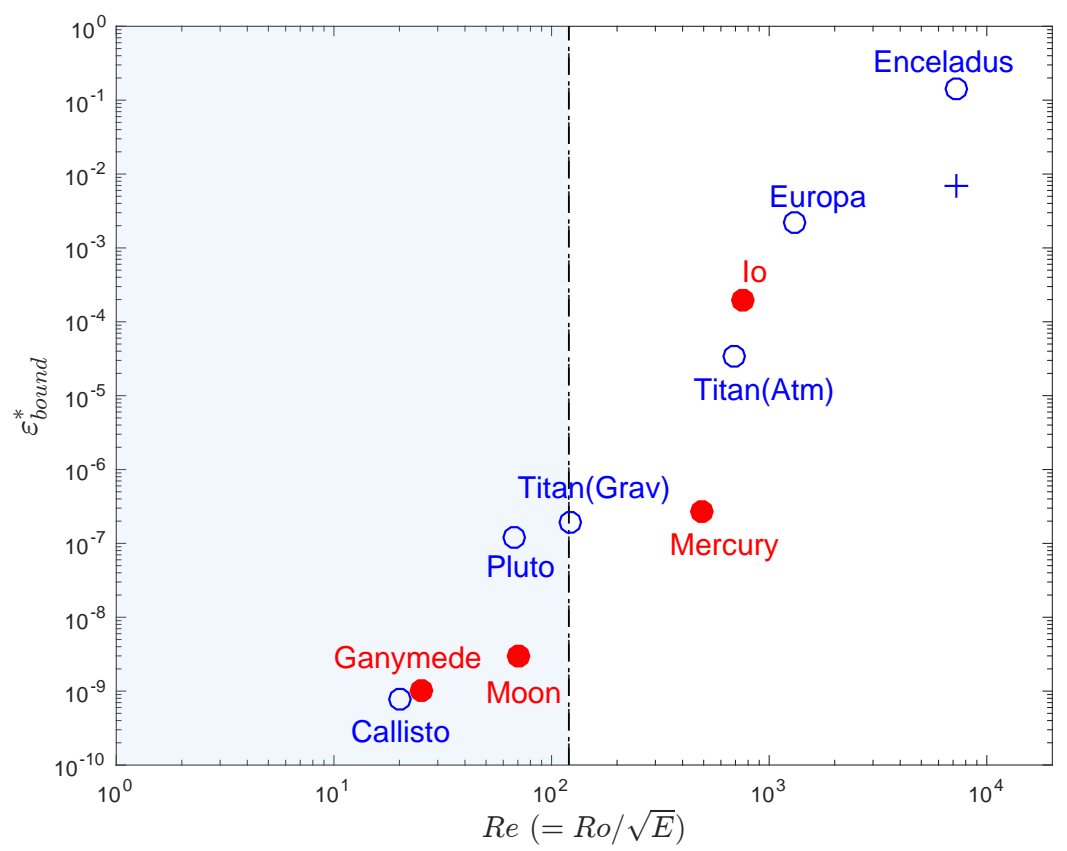

Figure 2: Bounds on the librational dissipation rate per unit surface area (units $W / m^{2}$ ) calculated for the bodies whose data is in table 1 versus the Reynolds number $R e:=R o / \sqrt{E}$ as defined in 25]. Subsurface oceans (of liquid water) are shown as blue empty circles and molten iron cores are shown as red filled circles. The blue cross marks the measured value of the flux per unit area for Enceladus [20]. The critical Reynolds number for boundary turbulence due to libration has been estimated as $R e_{c r i t}=120$ [25] so the (un)shaded region indicates (turbulent) laminar librational flow responses.

For the other satellites inferred to possess subsurface oceans or molten cores along with Mercury, the corresponding librational dissipation rate upper bounds are shown in figure 2 (using the data from table 1). Titan, Io, Europa, Mercury and particularly Enceladus all lie well within the region of Reynolds number found experimentally by 25 to show boundary turbulence. As a result, one can expect that the upper bound derived above will be a good predictor for the librational dissipation rate in these bodies modulo an appropriate renormalisation of the numerical coefficient (argued above to be about 2.5 orders of magnitude). Conversely, Callisto, Ganymede, the Earth's moon and Pluto all 
lie within either the stable or boundary layer rolls regime found in 25] and so these bodies.

\section{Discussion}

The upper bound on the turbulent dissipation rate derived here, which is for a librating spherical shell where only the outer boundary librates, is

20 times greater than the measured value of the globally averaged flux of $7 \times 10^{-3} \mathrm{~W} / \mathrm{m}^{2}$ [20]. Building in the renormalisation factor of 2.5 orders of magnitude $(\approx 300)$ already discussed, would mean that the predicted dissipation rate for librationally-drived turbulence in a spherical shell is then about 15 times too small. On the face of it, this looks a negative result but actually is not given that the ice crust on Enceladus will neither be perfectly smooth nor spherical. The same tidal effects which cause the libration will distort the crust to be dominantly elliptical in cross-section and then the flow is known to be even more prone to instability [39, 30] and experience dramatically enhanced turbulent dissipation (e.g. [30, 40, 41]). Roughness of the ice crust will also increase the librational driving of the subsurface ocean given the effect in other boundary-driven flows (e.g [42]) and provides a means by which locally-enhanced heating may occur. In particular, the ice crust could have a particularly rough inner surface near Enceladus's south pole where the surface heat flux is observed to be peaked.

205 Both roughness and tidal distortion crucially influence the fluid mechanics because then the librating ice crust will be inertially rather than just viscously coupled to the subsurface ocean through pressure forces which push the fluid back and forth as it librates. Ideally, these important effects should have been included in the mathematical bounding analysis presented here but, unfortunately, it is currently unknown how to treat boundary-driven flows where the boundary actually pushes the fluid around. Given this, the strategy pursued here instead has been to demonstrate how much dissipation can be generated 
in the most benign situation and then argue that the excluded effects can only dramatically increase the librational heating in the subsurface ocean beyond that observed. In particular, it doesn't take much to imagine that the presence of both roughness and tidal distortion in the outer ice crust could magnify the dissipation rate in the subsurface ocean by at least a factor of 15 to explain the observed heat flux emanating from Enceladus.

Our conclusion is then that indirect tidal stirring of the subsurface ocean via tidally-driven boundary motion of the ice crust is a viable process to generate the heat flux observed emanating from Enceladus. This complements the conclusion drawn in the opposite end-member scenario where the ice crust is ignored and the ocean tides are driven directly [19, 20, 21. Taken together, these end-member scenarios suggest that tidal forcing can heat Enceladus's ocean suf225 ficiently in the more complicated, realistic situation where the ice crust, ocean and solid core are mutually interacting under tidal forces.

The theoretical analysis presented here, which assesses the limits of the turbulent state directly, complements all the experimental and numerical work currently being done to understand mechanistically the fluid flows driven by precession and tides (e.g. see the review [43]). In the broader context, the general fact that small mechanical forcing can drive a significant (turbulent) response in a body of rotating fluid is currently not well known in the earth and space community (again see [4]]). Here we have studied libration following earlier work on precessing systems [34 to emphasize what is possible and 235 either seen or hinted at in experiments $33,30,40,41,44$. What is needed now is detailed numerical computations at low enough Ekman numbers to validate the scalings predicted here and data to calibrate the numerical prefactor of the bound so it can be used as a predictive estimate. If these work out, given the paucity of alternatives, it could be that Enceladus will do for the idea of tidal heating of fluids what Io did for tidal heating of viscoelastic solids nearly 40 years ago. 
Acknowledgements. We are grateful to Jerome Noir, Robert Tyler and an anonymous referee for insightful comments which helped considerably improve this manuscript and Jerome Noir for generating figure 1.

\section{Appendix A. Detailed bounding calculation}

Here the methods used to bound each of the terms in $(9)$ are detailed. The first term (1) is bounded as follows

$$
\begin{aligned}
\mid\left\langle\frac{\dot{\gamma}}{\mathcal{V}} \frac{1}{\ell} \int_{1-\ell}^{1} \int_{r^{\prime}}^{1} \oint\right. & \left.\boldsymbol{u} \cdot \hat{\boldsymbol{z}} \times \boldsymbol{r} d S d r d r^{\prime}\right\rangle|=|\left\langle\frac{\dot{\gamma}}{\mathcal{V}} \frac{1}{\ell} \int_{1-\ell}^{1} \int_{r^{\prime}}^{1} \int_{0}^{2 \pi} \int_{0}^{\pi} w r^{3} \sin ^{2} \theta d \theta d \phi d r d r^{\prime}\right\rangle \mid \\
\leqslant & \frac{R o f^{*}}{\ell \mathcal{V}} \int_{1-\ell}^{1} \int_{r^{\prime}}^{1} \int_{0}^{2 \pi} \int_{0}^{\pi}\langle|w|\rangle \sqrt{\sin \theta} d \theta d \phi d r d r^{\prime} \\
& \leqslant \frac{R o f^{*}}{\ell \mathcal{V}} \int_{1-\ell}^{1} \int_{r^{\prime}}^{1}\left\langle\int_{0}^{2 \pi} \int_{0}^{\pi} \sqrt{\sin \theta} \int_{r}^{1}\left|\frac{\partial w}{\partial r^{\prime \prime}}\right| d r^{\prime \prime} d \theta d \phi\right\rangle d r d r^{\prime} \\
\leqslant & \frac{R o f^{*}}{\ell \mathcal{V}} \int_{1-\ell}^{1} \int_{r^{\prime}}^{1}\left\langle\int_{0}^{2 \pi} \int_{0}^{\pi} \int_{r}^{1} r^{\prime \prime 2} \sin \theta\left|\frac{\partial w}{\partial r^{\prime \prime}}\right|^{2} d r^{\prime \prime} d \theta d \phi\right\rangle^{1 / 2} \\
& \times\left(\int_{0}^{2 \pi} \int_{0}^{\pi} \int_{r}^{1} \frac{1}{r^{\prime \prime 2}} d r^{\prime \prime} d \theta d \phi\right)^{1 / 2} d r d r^{\prime} . \\
\leqslant & \sqrt{\frac{2}{\mathcal{V}}} \pi R o f^{*} \frac{\sqrt{\ell^{3} \varepsilon}}{\sqrt{E}}
\end{aligned}
$$

where the second line uses the fact that $r^{3} \leqslant 1, \sin ^{3 / 2} \theta \leqslant 1$ and $|\dot{\gamma}| \leqslant R o f^{*}$.

The third line uses the Fundamental Theorem of Calculus to replace $w$ by the integral of its radial derivative away from $r=1$ where $w$ vanishes and then the integral is judiously split by the Cauchy-Schwartz inequality in the fourth and fifth lines. A final bound comes from extending the range of integration for $r^{\prime \prime}$ in the first integral (fourth line), bounding $\left|\partial w / \partial r^{\prime \prime}\right|^{2}$ by $|\nabla \boldsymbol{u}|^{2}$ and then bounding the second integrand (fifth line) (higher order terms in $\ell$ have been neglected as it is found that the optimal $\ell$ is much smaller than 1 ). 
The second term (2) is bounded as follows

$$
\begin{aligned}
\mid\left\langle\frac{\gamma}{\ell \mathcal{V}} \int_{1-\ell}^{1}\right. & \left.\left.\int_{0}^{2 \pi} \int_{0}^{\pi} r^{\prime 3} u w\right|_{r=r^{\prime}} \sin ^{2} \theta d \theta d \phi d r^{\prime}\right\rangle \mid \\
& \leqslant\left.\frac{R o}{\ell \mathcal{V}} \int_{1-\ell}^{1} \int_{0}^{2 \pi} \int_{0}^{\pi}\langle|u||w|\rangle\right|_{r=r^{\prime}} \sin \theta d \theta d \phi d r^{\prime} \\
& \leqslant \frac{R o}{\ell \mathcal{V}} \int_{1-\ell}^{1} \int_{0}^{2 \pi} \int_{0}^{\pi}\left\langle\int_{r^{\prime}}^{1}\left|\frac{\partial u}{\partial r}\right| d r \int_{r^{\prime}}^{1}\left|\frac{\partial w}{\partial r}\right| d r\right\rangle \sin \theta d \theta d \phi d r^{\prime} \\
& \leqslant \frac{R o}{\ell \mathcal{V}} \int_{1-\ell}^{1} \int_{r^{\prime}}^{1} \frac{1}{r^{2}} d r \int_{0}^{2 \pi} \int_{0}^{\pi}\left\langle\left(\int_{r^{\prime}}^{1} r^{2}\left|\frac{\partial u}{\partial r}\right|^{2} d r\right)^{1 / 2} \times\left(\int_{r^{\prime}}^{1} r^{2}\left|\frac{\partial w}{\partial r}\right|^{2} d r\right)^{1 / 2}\right\rangle \sin \theta d \theta d \phi d r^{\prime} \\
& \leqslant \frac{R o}{2 \ell \mathcal{V}} \int_{1-\ell}^{1} \int_{r^{\prime}}^{1} \frac{1}{r^{2}} d r \int_{0}^{2 \pi} \int_{0}^{\pi}\left\langle\int_{r^{\prime}}^{1} r^{2}\left(\left|\frac{\partial u}{\partial r}\right|^{2}+\left|\frac{\partial w}{\partial r}\right|^{2}\right) d r\right\rangle \sin \theta d \theta d \phi d r^{\prime} \\
& \leqslant \frac{1}{2} R o \ell \frac{\epsilon}{E}
\end{aligned}
$$

where $\gamma$ is bounded by its maximum, $R o, r^{\prime 3} \leqslant 1$ and $\sin \theta \leqslant 1$ in the second line. The fundamental theorem of calculus is used twice to replace $u$ and $w$ by integrals of their radial derivatives in the third line before the Cauchy-Schwartz inequality is used to split the integrals up. The inequality, $2 a b \leqslant a^{2}+b^{2}$, is now used in the fourth line. Thereafter, the rest of the terms of $|\nabla \boldsymbol{u}|^{2}$ are added to the second integrand and the range of integration extended to turn this into the dissipation rate expression and we bound the first integrand by its maximum (again higher order terms in $l$ have been neglected).

The third term (3) can be bounded by simply noting that $\sin \theta \leqslant 1$ and $\cos \theta \leqslant 1$ and following similar steps to those used for the first term (1). This leads to a final bound on (3) of

$$
4 \sqrt{\frac{2}{\mathcal{V}}} \pi R o(1+R o) \frac{\sqrt{\ell^{3} \epsilon}}{\sqrt{E}}
$$

The fourth term (4) is also bounded in the same fashion as (1) but without the step using the fundamental theorem of calculus, leading to a final bound on 
(4) of

$$
\frac{2}{\sqrt{\mathcal{V}}} \pi R o \frac{\sqrt{E \varepsilon}}{\sqrt{\ell}}
$$

\section{References}

[1] F. Nimmo, R. Pappalardo, Ocean worlds in the outer solar system,

(1) Journal of Geophysical Research 121 (2016) 1378-1399. doi:10.1002/ 275 2016JE005081.

[2] P. C. Thomas, R. Tajeddine, M. S. Tiscareno, J. A. Burns, J. Joseph, T. J. Loredo, P. Helfenstein, C. Porco, Enceladus's measured physical libration requires a global subsurface ocean, Icarus 264 (2016) 37-47. doi:10.1016/ j.icarus.2015.08.037.

[3] H. Hussmann, F. Sohl, T. Spohn, Subsurface oceans and deep interiors of medium-sized outer planet satellites and large trans-Neptunian objects, Icarus 185 (2006) 258-273. doi:10.1016/j.icarus.2006.06.005.

[4] J. Meyer, J. Wisdom, Tidal heating in Enceladus, Icarus 188 (2007) 535539.

[5] J. H. Roberts, F. Nimmo, Tidal heating and the long-term stability of a subsurface ocean on Enceladus, Icarus 194 (2008) 675-689. doi:10.1016/ j.icarus.2007.11.010.

[6] B. J. Travis, G. Schubert, Keeping Enceladus warm, Icarus 250 (2015) 32-42. doi:10.1016/j.icaurs.2014.11.017.

[7] C. J. A. Howett, J. R. Spencer, J. Pearl, M. Segura, High heat flow from Enceladus' south polar region measured using $10-600 \mathrm{~cm}^{-1}$ Cassini/cirs data, J. Geophys. Res. (Planets) 116.

[8] G. Schubert, J. D. Anderson, B. J. Travis, Enceladus: Present internal structure and differentiation by early and long-term radiogenic heating, Icarus 188 (2007) 345-355. doi:10.1016/j.icarus.2006.12.012. 
[9] J. R. Spencer, F. Nimmo, Enceladus: An active ice world in the saturn system, Annual Review of Earth and Planetary Sciences 41 (2013) 693717. doi:10.1146/annurev-earth-050212-124025.

[10] S. J. Peale, P. Cassen, R. T. Reynolds, Melting of Io by tidal dissipation, Science 203 (1979) 892-894.

[11] L. A. Morabito, S. P. Synnott, P. N. Kupferman, S. A. Collins, Discovery of currently active extraterrestrial volcanism, Science 204 (1979) 972.

[12] S. J. Peale, P. Cassen, R. T. Reynolds, Tidal dissipation, orbital evolution and the nature of Saturn's inner satellites, Icarus 43 (1980) 65-72.

[13] C. F. Yoder, Tidal friction and Enceladus's anomalous surface, EOS 62 (1981) 939.

[14] J. P. Poirier, L. Boloh, P. Chambon, Tidal dissipation in small viscoelastic ice moons: The case of Enceladus, Icarus 55 (1983) 218-230.

[15] M. N. Ross, G. Schubert, Viscoelastic models of tidal heating in Enceladus, Icarus 78 (1989) 90-101.

[16] G. Choblet, G. Tobie, C. Sotin, M. Behounkova, O. Cadek, F. Postberg, O. Soucek, Powering prolonged hydrothermal activity inside Enceladus, Nature Astronomydoi:10.1038/s41550-017-0289-8.

[17] M. Behounkova, G. Tobie, G. Choblet, O. Cadek, Impact of tidal heating on the onset of convection in Enceladus's ice shell, Icarus 226 (2013) 898-904. doi:10.1016/j.icarus.2013.06.033

[18] R. H. Tyler, Strong ocean tidal flow and heating on moons of the outer planets, Nature 456 (2008) 770-772.

[19] R. H. Tyler, Ocean tides heat Enceladus, Geophys. Res. Lett. 36 (2009) L15205. 
[20] R. H. Tyler, Tidal dynamical considerations constrain the state of an ocean on Enceladus, Icarus 211 (2011) 770-779. doi:10.1016/j.icarus.2010. 10.007

[21] R. H. Tyler, Comparative estimates of the heat generated by ocean tides on icy satellites in the outer solar system, Icarus 243 (2014) 358-385. doi: $10.1016 / j$. icarus.2014.08.037.

[22] I. Matsuymama, Tidal dissipation in the oceans of icy satellites, Icarus 242 (2014) 11-18.

[23] H. C. F. C. Hay, I. Matsuyama, Numericaly modelling tidal dissipation with bottom drag in the oceans of Titan and Enceladus, Icarus 281 (2017) 342-356. doi:10.1016/j.icarus.2016.09.022.

[24] E. M. A. Chen, F. Nimmo, G. A. Glatzmaier, Tidal heating in icy satellite oceans, Icarus 229 (2014) 11-30.

[25] J. Noir, F. Hemmerlin, J. Eict, S. M. Baca, J. M. Aurnou, An experimental and numerical study of librationally driven flow in planetary cores and subsurface oceans, Physics of the Earth and Planetary Interiors 173 (2009) 141-152. doi:10.1016/j.pepi.2008.11.012

[26] M. A. Calkins, J. Noir, J. D. Eldredge, J. M. Aurnou, Axisymmetric simulations of libration-driven fluid dynamics in a spherical shell geometry, Physics of Fluids 72 (2010) 086602. doi:10.1063/1.3475817.

[27] A. Sauret, D. Cebron, C. Morize, M. Le Bars, Experimental and numerical study of mean zonal flows generated by librations of a rotating spherical cavity, Journal of Fluid Mechanics 662 (2010) 260-268. doi:10.1017/ S0022112010004052

[28] D. Cebron, M. L. Bars, J. Noir, J. M. Aurnou, Libration driven elliptical instability, Physics of Fluids 24 (2012) 061703. 
[29] J. Noir, D. Cebron, M. L. Bars, A. Sauret, J. M. Aurnou, Experimental study of libration-driven zonal flows in non-axisymmetric containers, Physics of Earth and Planetary Interiors 204 (2012) 1-10.

[30] A. M. Grannan, M. Le Bars, D. Cébron, J. Aurnou, Experimental study of global-scale turbulence in a librating ellipsoid, Physics of Fluids 26 (2014) 126601. doi:10.1063/1.4903003.

[31] A. Sauret, D. Cebron, M. Le Bars, Spontaneous generation of inertial waves from boundary turbulence in a librating sphere, Journal of Fluid Mechanics 728 (2013) R5. doi:10.1017/jfm.2013.320

[32] R. R. Kerswell, Secondary instabilities in rapidly rotating fluids: inertial wave breakdown, Journal of Fluid Mechanics 382 (1999) 283-306.

[33] W. V. R. Malkus, Precession of the Earth as the cause of geomagnetism, Science 160 (1968) 777.

[34] R. R. Kerswell, Upper bounds on the energy dissipation in turbulent precession, Journal of Fluid Mechanics 321 (1996) 335-370. doi:10.1017/ S0022112096007756.

[35] C. E. Ozdemir, T.-J. Hsu, S. Balachandar, Direct numerical simulations of transition and turbulence in smooth-walled Stokes boundary layer, Phys. Fluids 26 (2014) 045108.

[36] C. Seis, Scaling bounds on dissipation in turbulent flows, Journal of Fluid Mechanics 777 (2015) 591-603. doi:10.1017/jfm.2015.384.

[37] O. Cadek, C. Tobie, T. Van Hoolst, M. Masse, G. Choblet, A. Lefevre, G. Mitri, R. Baland, M. Behounkova, O. Bourgeois, A. Trinh, Enceladus's internal ocean and ice shell constrained from Cassini gravity, shape and

口 libration data, Geophysical Research Letters 43 (2016) 5653-5660. doi: 10.1002/2016GL068634. 
[38] R. L. Comstock, B. G. Bills, A solar system survey of forced librations

n in longitude, Journal of Geophysical Research 108(E9) (2003) 5100. doi:

$375 \quad 10,1029 / 2003 J E 002100$

[39] R. R. Kerswell, W. V. R. Malkus, Tidal instability as the source for Io's magnetic signature, Geophysical Research Letters 25 (1998) 603-606. doi: 10.1029/98GL00237.

[40] B. Favier, A. M. Grannan, M. Le Bars, J. M. Aurnou, Generation and maintenance of bulk turbulence by libration-driven elliptical instability, Physics of Fluids 27 (2015) 066601. doi:10.1063/1.4922085.

[41] D. Lemasquerier, A. M. Grannan, J. Vidal, D. Cebron, B. Favier, M. L. Bars, J. M. Aurnou, Libration-driven flows in ellipsoidal shells, J. Geophys. Res. Planets 122 (2017) 1926-1950. doi:10.1002/2017JE005340.

${ }_{385}$ [42] T. H. van den Berg, C. R. Doering, D. Lohse, D. P. Lathrop, Smooth and rough boundaries in turbulent Taylor-Couette flow, Phys. Rev. E. 68 (2003) 036307.

[43] M. Le Bars, D. Cébron, P. L. Gal, Flows driven by libration, precession, 1 and tides, Annual Review of Fluid Mechanics 47 (2015) 163-193. doi: 10.1146/annurev-fluid-010814-014556.

[44] W. V. R. Malkus, An experimental study of global instabilities due to the tidal (elliptical) distortion of a rotating elastic cylinder, Geophysical and Astrophysical Fluid Dynamics 48 (1989) 123-134. 